\title{
Manejo de secuelas de defectos del esmalte en paciente con Síndrome Muenke: Reporte de caso
}

\section{Resumen}

El síndrome Muenke se caracteriza por retraso del desarrollo y pérdida auditiva neurosensorial. Personas que presentan síndromes podrían presentar defectos del desarrollo del esmalte. Sin embargo, en personas con síndrome Muenke no existe evidencia sobre la presencia y el manejo de las secuelas de estos defectos. Objetivo: Describir el manejo de secuelas de los defectos del desarrollo del esmalte en dientes primarios con un procedimiento odontológico integral en sala de operaciones de paciente con síndrome Muenke. Caso Clínico: Paciente de 2 años de edad, sexo masculino, con diagnóstico sistémico de Síndrome Muenke y diagnóstico odontológico: fracturas post eruptivas del defecto del esmalte extensión I ( $<1 / 3$ del diente) y II (de $1 / 3$ a $2 / 3$ del diente) en los dientes anteroinferiores y

\author{
Anahí Francisca Huamán Ochoa, ${ }^{1}$ \\ Gilmer Torres Ramos, ${ }^{1}$ \\ María Medrano Hernández, ${ }^{1}$ \\ Roxana Patricia López Ramos, ${ }^{2}$
}

fracturas post eruptivas del defecto del esmalte extensión III (> 2/3 del diente) de los dientes 62 y 52. El manejo clínico de estas secuelas, realizadas en sala de operaciones, consistió en: carillas de resina compuestas fotopolimerizable que permitieron restaurar las fracturas post eruptivas de los dientes antero inferiores; y los dientes 62 y 52 fueron rehabilitadas con coronas de resina compuesta fotopolimerizable a mano alzada. Conclusión: El manejo de las secuelas de los defectos de desarrollo del esmalte, en paciente con Síndrome Muenke, se consideró clínicamente positivo; pues después de 6 meses los dientes tratados no presentaron dolor espontáneo, ni se observó fistulas o absceso, ni movilidad dentaria.

Palabras clave: esmalte dental, hipoplasia del esmalte dental, síndrome, craneosinostosis (DeCS). 
Caso clinico

\section{Tratamento de sequelas de defeitos do esmalte em patiente com síndrome Muenke: Relato de caso}

\section{Resumo}

O síndrome Muenke é caracterizado por atraso no desenvolvimento e perda auditiva neurossensorial. Pessoas com síndrome podem ter defeitos de desenvolvimento do esmalte. No entanto, em pessoas com síndrome Muenke não há evidências sobre o manejo das sequelas desses defeitos. Objetivo: Descrever o manejo das sequelas de defeitos de desenvolvimento do esmalte em dentes decíduos com um procedimento odontológico abrangente na sala de cirurgia de um paciente com síndrome Muenke. Caso Clínico: Paciente masculino de 2 anos com diagnóstico sistêmico de Síndrome Muenke e diagnóstico dentário: Fraturas pós-eruptivas da extensão do defeito de esmalte I ( $<1 / 3$ do dente) e II (1/ 3 - 2/3 do dente) nos dentes anteriores inferiores. e fraturas pós-eruptivas da extensão do defeito de esmalte III ( $>2 / 3$ do dente) dos dentes 62 e 52 . Os tratamentos realizados foram com resina compostas fotopolimerizáveis que permitiu restaurar as fraturas pós-eruptivas da extensão I e II do defeito do esmalte dos dentes anteriores inferiores. Nos dentes 62 e 52 com fraturas pós-eruptivas do defeito de esmalte de extensão III. foram restauradas com coroas de resina compostas fotopolimerizáveis à mão libre. Conclusão: Os tratamentos foi considerado clinicamente positivo em paciente com Síndrome de Muenke; porque após 6 meses os dentes tratados não apresentavam dor espontânea, nem fístulas ou abscessos, nem mobilidade dentária.

Palabra-chave: Esmalte dentario, hipoplasia do esmalte dentario, síndrome, craniossinostose

Case report

\section{Management of Sequelae of the enamel defects in patient with Muenke syndrome: Case report}

\section{Abstract}

Muenke syndrome is characterized by developmental delay and sensorineural hearing loss. People with a syndrome may have enamel development defects. However, in people with Muenke syndrome, there is no evidence on the management of the sequelae of these defects. Objective: To describe the management of sequelae of enamel development defects in the operating room with a comprehensive dental procedure in primary teeth in a single sesión. Clinical case: 2 -year -old, male patient with medical diagnosis: Muenke Syndrome and dental diagnosis: Post eruptive fractures of the enamel defect extensión $I(<1 / 3$ of the tooth) and II (at least $1 / 3$ but less than $2 / 3$ of the affected tooth) in teeth $63,73,72,71,81,82,83$ and post- eruptive of the extensión III (more than $2 / 3$ of the tooth is affected) enamel defect of teeth 62 and 52, treatment consisted in: veneers that allowed to restore the post eruptive fractures of lower anterior teeth and composite 
crowns in teeth 62 and 52. Conclusion: The treatment was considered positive clinically since after six months in the patient with Muenke Sindrome; teeth did not present spontaneous pain, neither

\section{Introducción}

La craneosinostosis es un defecto de nacimiento definido como el cierre prematuro de uno o más suturas craneales. ${ }^{1}$ El síndrome de Muenke está asociado con una mutación en el gen del receptor 3 del factor de crecimiento de fibroblastos. ${ }^{2}$ Afecta 1 de cada 30.000 nacidos vivos y representa el $24 \%$ de la totalidad de defectos craneales, su principal característica es que puede ser bicoronal y unilateral ${ }^{3}$ en la mayoría de los casos hay una pérdida auditiva neurosensorial. ${ }^{4} \mathrm{El}$ diagnóstico neuropsiquiátrico más común es el retraso en el desarrollo, también se encontró pacientes con autismo, déficit de atención e hiperactividad. ${ }^{5}$

En el Perú, se introdujo la ley que declara de interés nacional y preferente atención al tratamiento de personas que padecen enfermedades raras o huérfanas, dentro de este listado se ubica el síndrome de Muenke. ${ }^{6}$ El tratamiento de este síndrome es multidisciplinario con otros servicios como genética, cirugía de cabeza y cuello, otorrinolaringología, neurología y odontología. Los problemas bucales, que se pueden presentar más comúnmente, son la maloclusión clase III debido a hipoplasia del tercio medio de la cara. ${ }^{7}$

El desarrollo de la dentición primaria comienza aproximadamente en la semana 12 del embarazo con la formación y fistulas or abscess were observed, nor dental mobility.

Key words: dental enamel, dental enamel hypoplasia, syndrome, craniosynostoses.

maduración del esmalte, a partir de la lámina dental que se origina en las células epiteliales del ectodermo oral, se forma 20 yemas o brotes de dientes que corresponderán a los dientes primarios. ${ }^{8}$ Entonces un hecho importante que se ha visto es que la yema del diente en desarrollo es sensible a una amplia gama de trastornos sistémicos y en particular en el esmalte. Los estudios poblacionales sobre el defecto del desarrollo del esmalte en la dentición primaria son escasos y ofrecen resultados inconsistentes con tasas de prevalencia que van desde 24 al $81 \%{ }^{9}$ La etiología apunta a factores sistémicos y factores ambientales dentro de ellos se encuentran defectos cualitativos y cuantitativos. ${ }^{10}$ Otros factores etiológicos son que los recién nacidos prematuros tienen dientes menos sanos que aquellos que nacieron a término ${ }^{11}$ y los niños con bajo peso tienen más probabilidades de presentar algún tipo de hipoplasia. ${ }^{12}$ Los defectos del esmalte resultante de la alteración de la amelogénesis hacen que la superficie dental sea defectuosa y pueda proporcionar un sitio adecuado para la adhesión y colonización de bacterias cariogénicas, ${ }^{13,14}$ por tal motivo se ha encontrado una asociación entre la caries dental y los defectos del esmalte en la primera infancia. ${ }^{15}$ Algunos trastornos sistémicos influyen en el desarrollo de los tejidos dentales como el esmalte y la dentina. ${ }^{16}$ Aún no se ha encontrado evidencia valida que asocie el síndrome de Muenke con defectos del esmalte. 
Los tratamientos odontológicos para el manejo de las secuelas de los defectos del esmalte van desde una microabrasión del esmalte, carilla de resina, el uso de resinas infiltrantes, utilización de barnices fluorados, también el uso de fosfopéptido de caseína fosfato de calcio amorfo (CPPACP) para fracturas post eruptivas del defecto del esmalte extensión I (cuando está afectado menos de $1 / 3$ del diente) y II (cuando al menos $1 / 3$ pero menos de $2 / 3$ del diente afectado) y cuando se presentan fracturas post eruptivas del defecto del esmalte extensión III ( afecta más de 2/3 del diente) los tratamientos son corona de acetato y pulpectomía, ${ }^{17}$ estos tratamientos pueden ser los más efectivos. Por lo tanto, el objetivo del presente trabajo fue describir el manejo de secuelas de los defectos del desarrollo del esmalte en dientes primarios con un procedimiento odontológico integral en sala de operaciones.

\section{Reporte de Caso}

Paciente de 2 años 2 meses de edad, sexo masculino, procedente de la ciudad de Lima, que acude al Servicio de Odontología del Instituto Nacional de Salud del Niño -San Borja. Madre refiere que su hijo no puede comer porque le duele los dientes y acude al servicio de odontología para tratamiento integral odontológico. Además, no refiere ningún antecedente patológico al igual que el padre, familia nuclear y con un nivel socioeconómico medio.

Antecedentes prenatales: pesó al nacer $3,330 \mathrm{~kg}$, parto distócico por circular doble, refiere cinco controles prenatales. Antecedentes post natales: Lactancia mixta hasta los 2 años. Antecedentes patológicos: Síndrome de Muenke, epistaxis y anemia microcítica. Al examen físico general presentó: Saturación de oxígeno del 99\%, frecuencia cardiaca de 95 por minuto, frecuencia respiratoria de 22 por minuto; peso de 16,300 kg; piel tibia, húmeda y elástica. A la palpación se encuentra tejido celular subcutáneo de volumen regular, sin edemas. Lo ojos reactivos a la luz y acomodación relativo a pupilas, fosas nasales permeables, faringe no congestiva, conducto auditivo externo permeable.

En el examen clínico estomatológico extrabucal se evidenció un paciente macrocéfalo, frente prominente e hipoplasia del tercio medio. Al examen estomatológico intrabucal presentó dentición primaria, inflamación generalizadas de encías, defectos del esmalte en dientes $63,73,72,71,81,82,83$; dolor espontáneo en los dientes 52 y 62; presentó mala higiene oral. Paciente no colaborador llora y grita al momento de examinarlo.

El diagnóstico estomatológico fue: Gingivitis marginal generalizada asociada a placa bacteriana, fracturas dentales post eruptivas de los defectos del esmalte extensión I y II en los dientes 63, 73, 72, $71,81,82$ y 83 y fracturas post eruptivas de los defectos del esmalte de extensión III de los dientes 62 y 52; además dientes 52 y 62 presentaron pulpitis irreversible. (Figura 1). Oclusión: mordida abierta, caries dental y conducta: comportamiento definitivamente negativo, según Frankl.

Previo tratamiento estomatológico integral se realizó fisioterapia oral. El plan de tratamiento consistió: pulpectomías en los dientes 52 y 62, coronas de resina 


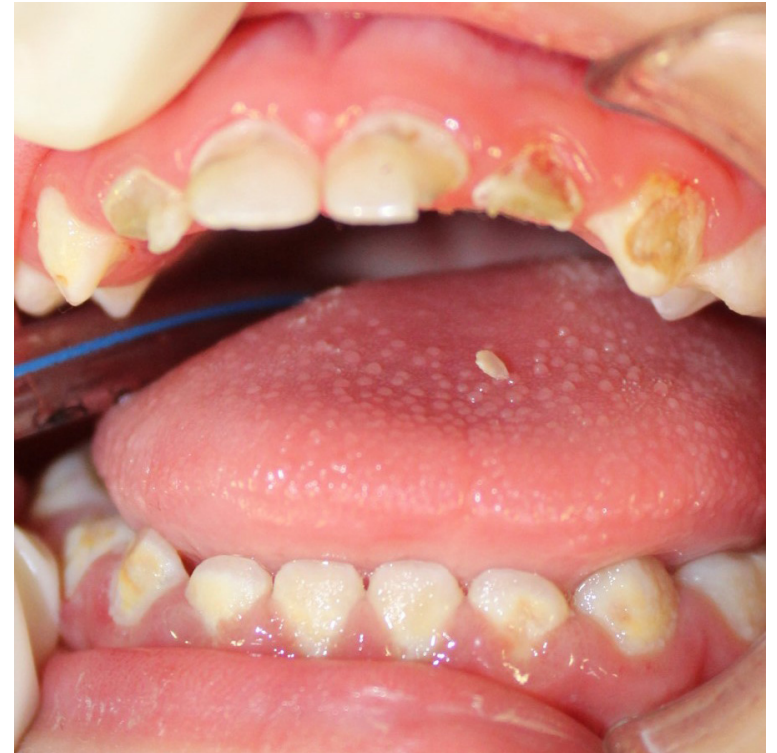

Figura 1: Presentación de las secuelas de los defectos del esmalte, fracturas post eruptivas extensiones III en los dientes 62, 52 y fracturas post eruptivas extensión I y II en las piezas 63, 73, $72,71,81,82,83$.

microhíbrida fotopolimerizable a mano alzada: 52 y 62, carillas de resina microhíbrida fotopolimerizable: 73, 72,
72, 81, 82 y 83; como mantenimiento post operatorio se recomendó uso de pasta dental 1000 ppm del tamaño de un arroz tres veces al día.

Se explicó a la madre sobre el diagnóstico estomatológico de su hijo y lo que implicaba el tratamiento integral estomatológico que sería realizado en sala de operaciones por las múltiples secuelas de defectos del esmalte en los dientes, además a la nula cooperación del niño y condición sistémica. Se obtuvo el consentimiento informado firmado de la madre, según las normativas del comité ética de la institución hospitalaria.

Previo al tratamiento odontológico integral en sala de operaciones, se realizaron los exámenes e interconsultas pertinentes, para evaluar el estado físico y obtener las sugerencias correspondientes. Los exámenes fueron de laboratorio (Tabla 1) y de radiografía del tórax.

Tabla 1: Resultados de los exámenes de laboratorio.

\begin{tabular}{|l|c|c|}
\hline Examenes de laboratorio & Valores del paciente & Valores normales \\
\hline Hematocrito & $29,9 \%$ & $35-44 \%$ \\
\hline Leucocito & $7.590 / \mathrm{mm}^{3}$ & $7.000-13000 / \mathrm{mm}^{3}$ \\
\hline Eosinófilos & $1 \%$ & $1-4 \%$ \\
\hline Linfocitos & $20 \%$ & $20-40 \%$ \\
\hline Plaquetas & $321000 / \mathrm{mm}^{3}$ & $150.000-450.000 / \mathrm{mm}^{3}$ \\
\hline Hemoglobina & $8,8 \mathrm{~g} / \mathrm{dl}$ & $12-15 \mathrm{~g} / \mathrm{dl}$ \\
\hline Glucosa & $83 \mathrm{mg} / \mathrm{dl}$ & $90-120 \mathrm{mg} / \mathrm{dl}$ \\
\hline Creatinina & $0,27 \mathrm{mg} / \mathrm{dl}$ & $0,7-1,2 \mathrm{mg} / \mathrm{dl}$ \\
\hline Tiempo de protrombina & $10,5 \mathrm{seg}$ & $10-15 \mathrm{seg}$ \\
\hline Tiempo parcial tromboplastina activada & $21 \mathrm{seg}$ & $20-30 \mathrm{seg}$ \\
\hline Examen de orina & No patológico & \\
\hline
\end{tabular}


Se solicitó interconsulta al servicio de anestesiología, el cual indico ASA II e interconsultaal servicio de pediatría quien evaluó el resultado de la hemoglobina $(8,8 \mathrm{~g} / \mathrm{dl})$ y diagnosticó anemia crónica por epistaxis y dio tratamiento con Ferranin. El pediatra refirió que, por la condición de los dientes, el paciente no se alimentaba adecuadamente; y sugirió la inmediata realización del tratamiento, de esa manera mejoraría su estado nutricional, a pesar de su nivel bajo de hemoglobina. Se tomó en cuenta que no se realizarían exodoncias solo tratamientos conservadores en los dientes del niño. También el pediatra refirió una interconsulta a hematología posterior al tratamiento odontológico en sala de operaciones, para el seguimiento de la hemoglobina. El único inconveniente fue realizar la radiografía panorámica y periapical, que por la edad del paciente dificultaba el procedimiento.

No se realizaron cambios en el plan de tratamiento, respetando la conservación de los dientes. Paciente con pronóstico favorable, ya que se trataron todos los dientes, no hubo pérdida de ninguna pieza dentaria y sobre todo por la concientización a la madre sobre el cuidado de la salud bucal de su hijo (Figura 2).

Se evaluó al paciente por medio de solo controles clínicos post operatorios, puesto que la paciente presentó nula colaboración a la toma de radiografías periapicales y panorámica. Estos controles fueron a los 7 días y a los 2 meses, no se observó desprendimientos

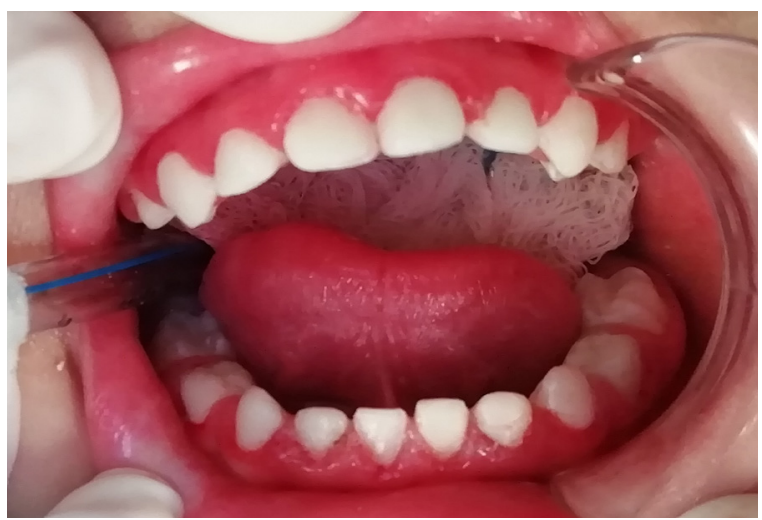

Figura 2: Rehabilitación de las fracturas post eruptivas de extensión I y II con carillas de resina compuesta fotopolimerizable en dientes 63, 73,

$72,71,81,82,83$ y en las fracturas post eruptivas de extensión III se realizaron pulpectomías en los dientes 52, 62, y con sus respectivas coronas de resina compuesta fotopolimerizable a mano alzada de las piezas 52,62 .

de restauraciones, presencia de las coronas de resina y carillas de resina en boca, nivel de higiene bucal buena. Madre del paciente refirió que su hijo no presentó ninguna molestia después del tratamiento, se alimenta con normalidad, ya no presenta dolor al momento de masticar, y se citó a madre para su segundo control en dos meses. En segundo control, madre refiere que no ha tenido ningún dolor ni molestia. Al examen clínico no se observó desprendimiento de restauraciones en los dientes, se observa una encía más rosada y menos sangrante, se vuelve a dar indicaciones de higiene bucal y se citó en 6 meses. En resumen, paciente después de sus controles no evidencia signos clínicos de inflamación. A la fecha no se suscitó algún evento adverso de parte de nuestro paciente (Figura 3). 


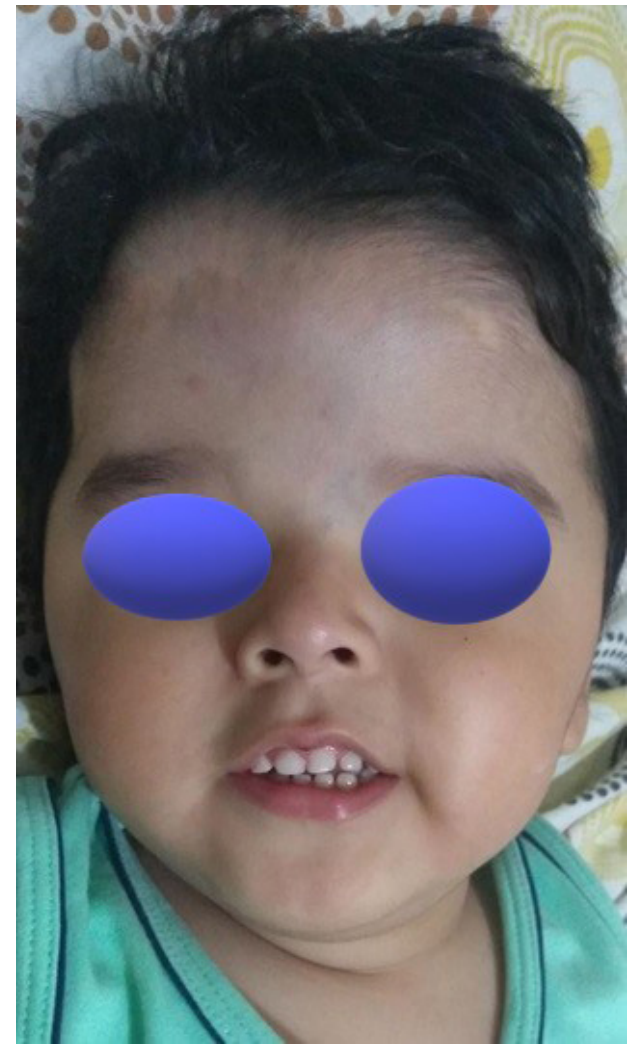

Figura 3: Control a los 6 meses.

\section{Discusión}

El presente trabajo describe el manejo de secuelas de los defectos del desarrollo del esmalte con un procedimiento odontológico integral en sala de operaciones en dientes primarios en una sola sesión de un paciente con Síndrome de Muenke.

El uso de anestesia general en niños exige los medios necesarios para un control adecuado de signos vitales, un personal entrenado que maneje el equipo necesario para solventar posibles emergencias, ${ }^{18}$ lo cual no sería posible en centros de salud o puestos de salud haciendo menos accesible a la población que viene del interior del país. El presente caso presentó varios dientes a ser restaurados, sumado a que era un paciente no colaborador y pues realizar dichos tratamientos en varias citas no era viable. Los tratamientos estomatológicos integrales que se realizaron al paciente fueron en sala de operaciones y realizados con los objetivos de prevenir infecciones odontogénicas, devolver la función masticatoria y también mejorar el aspecto estético del niño.

El manejo de las secuelas de los defectos del esmalte se realiza a través de una serie de tratamientos. Uno de estos tratamientos es la microabrasión pero que está contraindicado en pacientes que no cooperan, pacientes con sensibilidad dentinaria y no se recomienda en lesiones hipoplásicas profundas del esmalte. El presente caso fue de un niño no colaborador y las lesiones cariosas que presentaba eran profundas por ello fue descartado dicho tratamiento. Otro tratamiento de estas secuelas es con el uso de resinas infiltrantes, este es indicado para detener las caries incipientes del esmalte ${ }^{19}$ y lesiones de manchas blancas causadas por desmineralización del esmalte; ${ }^{20}$ no se realizó en el presente caso puesto que el paciente presentaba cavidades profundas no solo en la superficie del esmalte sino también en dentina.

Por otro lado, la técnica con coronas de acetato es fácil de ajustar y recortar, tiene una fácil adaptación al remanente dentario y su acabado deja una superficie lisa y brillante. ${ }^{21}$ En el presente caso, se realizaron coronas de resina a mano alzada para disminuir el tiempo operatorio que es limitado en sala de operaciones. Las restauraciones con resina compuesta fotopolimerizable fueron realizadas con una resina microhíbrida por contener partículas de 0,6 a 1,4 $\mu \mathrm{m}$ y $20 \mathrm{~nm} .{ }^{22}$ Estas resinas presentan clínicamente una buena 
adaptación marginal, color similar a la estructura dental, ${ }^{11}$ buena capacidad de pulido, baja presencia de caries recurrente, y baja sensibilidad postoperatoria, ${ }^{23}$ haciendo un material de elección para los diferentes tratamientos que se realizaron en el paciente como las carillas y coronas de resina a mano alzada. ${ }^{24}$ Tener en cuenta que la vida útil de las coronas y carillas de resina estarán muy ligadas a la higiene oral del paciente, ya que si no existe una buena higiene oral esto producirá inflamación gingival y la desadaptación de la corona de resina y carilla de resina. ${ }^{24}$

También es recomendado como tratamiento el uso de fosfopéptido de caseína- fosfato de calcio amorfo (CPP$\mathrm{ACP})$ que es un material innovador por la utilización de la caseína que es una proteína de la leche ${ }^{22}$ este material proporciona extra-protección a los dientes y ayuda a neutralizar los cambios de ácido de las bacterias acidogénicas en la placa. Sin embargo, su alto costo impide la accesibilidad del material para su uso en este caso. Es así que la utilización de flúor en barniz para remineralizar se ha convertido en uno de los métodos más usados para prevenir caries dental en dientes primarios, su efectividad, seguridad y facilidad en su aplicación lo hace un material sumamente usado por los odontólogos. ${ }^{22}$ Es por ello que, en el presente caso fue útil la aplicación de barniz fluorado debido a las ventajas que presenta y se utilizó en el paciente en la fase educativa antes de procedimiento odontológico y después para proteger la zona lingual de los dientes tratados con las carillas de resina.

\section{Conclusiones}

El manejo de las secuelas de los defectos de desarrollo se consideró clínicamente positivo, en el paciente con Sindrome Muenke, pues después de seis meses los dientes tratados no presentaron dolor espontáneo, tampoco se observó fistulas o absceso, ni movilidad dentaria. Los dientes que se restauraron con carillas y coronas de resina compuestas fotopolimerizable tampoco presentaron un desprendimiento en los controles. Siendo este tipo de manejo odontológico el adecuado proporcionándole una mejora en la calidad de vida del paciente.

\section{Conflictos de intereses}

Ninguno.

\section{Financiamiento}

Autofinanciado.

\section{Referencias bibliográficas}

1. Saavedra-Ontiveros MD, Morán-Barroso VF. II. Aspectos clínicos en craneosinostosis. Gac Med Mex. 2003;139(3):236-9.

2. Honnebier MB, Cabiling DS, Hetlinger M, McDonald-Mcginn DM, Zackai EH, Bartlett SP. The natural history of patients treated for FGFR3-associated (Muenke type) craniosynostosis. Plast Reconstr Surg. 2008;121(3):919-31.

3. Yasuda T, Nah HD, Laurita J, Kinumatsu T, Shibukawa Y, Shibutani T, et al. Muenke syndrome mutation, FgfR3 P244R, Causes TMJ Defects. J Dent Res. 2012; 91(7): 683-9

4. Mansour SL, Twigg SRF, Freeland RM, Wall SA, Li C, Wilkie AOM. Hearing loss in a mouse model of Muenke syndrome. Hum Mol Genet. 2009; 18(1):43- 5012. 
5. Murali CN, McDonald-McGinn DM, Wenger TL, McDougall C, Stroup BM, Sheppard SE, et al. Muenke syndrome: Medical and surgical comorbidities and long-term management. Am J Med Genet Part A. 2019; 179(8): 1442-50.

6. MINSA. Resolución Ministerial N 151-2014/MINSA. 2014;517400-6. Disponible en: ftp://ftp2.minsa. gob.pe/normaslegales/2014/RM151_2014_MINSA_EP.pdf

7. Samra F, Bauder AR, Swanson JW, Whitaker LA, Bartlett SP, Taylor JA. Assessing the midface in Muenke syndrome: A cephalometric analysis and review of the literature. J Plast Reconstr Aesthetic Surg. 2016; 69(9):1285-90

8. Suckling GW. Developmental defects of enamel--historical and present-day perspectives of their pathogenesis. Adv Dent Res. 1989; 3(2):87-94.

9. Ruschel HC, Vargas-Ferreira F, Tovo MF, Kramer PF, Feldens CA. Developmental defects of enamel in primary teeth: highly prevalent, unevenly distributed in the oral cavity and not associated with birth weight. Eur Arch Paediatr Dent. 2019; 20(3):241-248. doi:10.1007/s40368-018-0402-4.

10. Alshehhi A, Al Halabi M, Hussein I, Salami A, Hassan A, Kowash M. Enamel defects and caries prevalence in preterm children aged 5-10 years in Dubai. Libyan J Med. 2020 Dec;15(1):1705633. doi: 10.1080/19932820.2019.1705633. PMID: 31873070; PMCID: PMC6968668.

11. Schüler IM, Haberstroh S, Dawczynski K, Lehmann T, Heinrich-Weltzien R. Dental Caries and Developmental Defects of Enamel in the Primary Dentition of Preterm Infants: Case-Control Observational Study. Caries Res. 2018; 52(1-2):22-31.

12. Masumo R, Bårdsen A, Åstrøm AN. Developmental defects of enamel in primary teeth and association with early life course events: A study of 6 - 36 month old children in Manyara, Tanzania. BMC Oral Health. 2013; 13(1):1-11.

13. Caries EC. Early Childhood Caries: IAPD Bangkok Declaration. Int J Paediatr Dent.2019;29(3):384-6.

14. Alkhtib A, Ghanim A, Temple-Smith M, Messer LB, Pirotta M, Morgan M. Prevalence of early childhood caries and enamel defects in four and five-year old Qatari preschool children. BMC Oral Health. 2016; 16(1):1-7.

15. Caufield PW, Li Y, Bromage TG. Hypoplasia-associated severe early childhood caries a proposed definition. J Dent Res. 2012; 91(6):544-50

16. Atar M, Körperich EJ. Systemic disorders and their influence on the development of dental hard tissues: A literature review. J Dent. 2010; 38(4):296-306.

17. Ghanim A, Silva MJ, Elfrink MEC, Lygidakis NA, Mariño RJ, Weerheijm KL, et al. Molar incisor hypomineralisation $(\mathrm{MIH})$ training manual for clinical field surveys and practice. Eur Arch Paediatr Dent 2017; 18(4):225-42.

18. Del Patrocinio Vargas Román M, Rodríguez Bermudo S, Machuca Portillo G. Tratamiento odontológico bajo anestesia general: ¿Un procedimiento útil en el tercer milenio? (I). Med Oral. 2003; 8(2):129-35.

19. Kim S, Kim EY, Jeong TS, Kim JW. The evaluation of resin infiltration for masking labial enamel white spot lesions. Int J Paediatr Dent. 2011;21(4):241-8. 22.

20. Paris S, Schwendicke F, Keltsch J, Dörfer C, Meyer-Lueckel H. Masking of white spot lesions by resin infiltration in vitro. J Dent. 2013; 41 Suppl 5:e28-e34.

21. De Andrade AKMI, Duarte RM, Medeiros E Silva FDSC, Batista AUD, Lima KC, Pontual MLDA, et al. 30-Month randomised clinical trial to evaluate the clinical performance of a nanofill and a nanohybrid composite. J Dent. 2011; 39(1):8-1

22. Maas JRS, Junior IMF, Lodi CS, Delbem ACB. Differences in loosely bound fluoride formation and anticaries effect of resin-based fluoride varnishes. Int J Paediatr Dent.2013; 23(3):166-72.

23. De Andrade AKM, Duarte RM, Medeiros e Silva FDSC, Batista AUD, Lima KC, De Melo Monteiro GQ, et al. Resin composite class I restorations: A 54- month randomized clinical trial. Oper Dent. 2014; 39(6):58894.

24. Gaurav Kumar M AV. Crowns in Pediatric Dentistry: a Review. J Adv Med Dent Scie Res 2016; 4(2):41-46.

Recibido: 07/09/2020

Aceptado: 6/02/2021

Correspondencia: Roxana Patricia López Ramos, correo: roxana.lopez.r@upch.pe 